\title{
Energy Storage Device Application for Load Oscillations Damping in Isolated Power Systems
}

\author{
Natalya Kiryanova \\ Department of Automated Electrical Power Systems \\ Novosibirsk State Technical University \\ Novosibirsk, Russia \\ kiryanova-ng@ya.ru \\ Gleb Prankevich \\ Department of Automated Electrical Power Systems \\ Novosibirsk State Technical University \\ Novosibirsk, Russia \\ prankevich.gleb@mail.ru
}

\author{
Dmitry Baluev \\ Department of Automated Electrical Power Systems \\ Novosibirsk State Technical University \\ Novosibirsk, Russia \\ baluev.dy@gmail.com \\ Vyacheslav Zyryanov \\ Department of Automated Electrical Power Systems \\ Novosibirsk State Technical University \\ Novosibirsk, Russia \\ zvmov@ngs.ru
}

\begin{abstract}
In isolated power systems for energy-intensive consumers with changing power consumption, there is a need to investigate how different operating and consumption modes influence the system parameter and quality of electricity. This issue is relevant for power systems, which include energyintensive consumers with changing power consumption. The load diagram of mentioned consumers has stochastic nature with the average value of about $1 / 4-1 / 5$ of generator units rated power. Such operation mode adversely affects the specific fuel consumption of the generator. In addition, an abruptly variable load can cause (or enhance) low-frequency oscillations (LFO) in the power system. The energy storage system (ESS) is an effective tool for solving the problem of full or partial compensation of load fluctuations. This paper shows the research results of application of storage, based on a supercapacitor for lowfrequency oscillations damping in the isolated power system. Calculations are performed with a help of the MATLAB/Simulink software package. A technique for choosing ESS parameters is developed.
\end{abstract}

Keywords-abruptly variable load; energy storage system; isolated power system; power system stability; electricity quality; supercapacitor

\section{INTRODUCTION}

The development of energy storage technologies has already reached the level of practical implementation in the electric power industry. Some types of ESS allow one to effectively solve different tasks and problems in energy systems [1-4]. One of the problems is abruptly variable load. Energy-intensive consumers can produce a significant negative impact on the regime characteristics. And also, they can cause LFO in interconnected and in isolated power systems $[5,6]$.

Each generator is equipped with the automatic speed regulator and the automatic excitation regulator that have their own parameters and characteristics. The regulators react to any disturbance caused by a load changing. The reaction of regulators leads to the oscillating process and changes in regime parameters of generators due to their inertia and dead zones. There is always a possibility of an electromechanical resonance between the generator with its automatic control systems and the periodically changing load because the load is a constant source of disturbances. If load fluctuations are slow, frequency and voltage automatic regulation systems of the generator are able to provide a scheduled operating mode with the required accuracy. If frequency of load changing does not allow regulators to respond adequately to disturbances due to inertia, regulation is actually realized according to average values of rapidly varying power values (for the automatic speed regulator) and voltage (for the automatic excitation regulator). For a short-term power surge, the automatic speed regulator is actually unable to react effectively because the frequency does not have time to change noticeably in a short time interval of the disturbance.

\section{RANGE OF THE HIGHEST SENSITIVITY OF THE GENERATORS}

For each generator aggregate, there is a range of frequencies of periodic perturbations. In this range, all the inertia, dead zones and droop characteristics of regulators and control systems have a maximum affect. In this range, the quality of frequency and voltage regulation is the worst. It proceeds by the greatest deviations of these parameters from the desired values. It is actual for any type of power systems, but most important for autonomous systems. In such systems, the single-unit power of single consumers is comparable to the power of the energy system.

In order to determine the frequency range of load oscillations that lead to the largest deviations in regime parameters of main generator units, calculations of transients in the simplest system (generator unit and load) are performed. The load had constant ( 0.65 p.u.) and variable $(0.35$ p.u. $)$ components. The frequency of the variable component of the load was varying harmonically in the range from zero to infinity. 
The calculation experiment was performed by means of the MATLAB/Simulink software package for several types of generator units from the MATLAB/Simulink standard library.

Fig. 1 shows the dependence of the amplitude values of the frequency deviation of generators from the nominal value on the variation of the frequency of the harmonic component of the load. For each type of the generator that equipped with the typical automatic speed regulator, automatic excitation has its own frequency range of load oscillations. The greatest sensitivity of generators to disturbances and frequency deviations reaches the highest values within this range. The presence of harmonics from this range in the load diagram of the generator can lead to the appearance or amplification of already existing oscillations of the operating conditions of the power system (including LFOs that have frequencies in the same range).

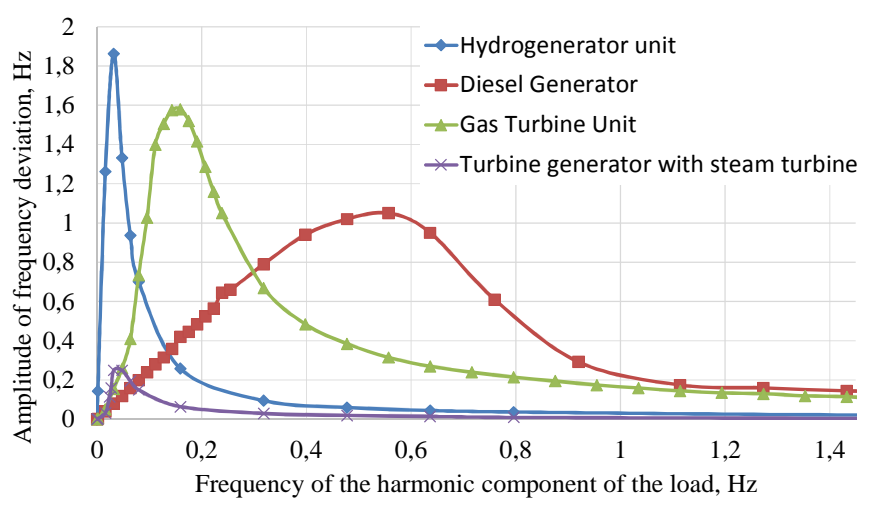

Fig. 1. Dependence of amplitude values of frequency deviation of generators on frequency of harmonic component of load

\section{ABRUPTLY VARIABLE LOAD}

For example, the consumers with a periodic or abruptly variable power consumption are consumers of the oil and gas industry (beam-pumping units), metallurgy and mechanical engineering (engines for mechanisms operating in a wide range of speeds with frequent starts and stops), electric arc furnaces, powerful lift and transport mechanisms and a number of others.

Fig. 3 shows a typical fragment of the load diagram of an autonomous power station of a floating crane (obtained from the field experiment) in the normal technological mode of operation when loose cargo is craned. The average load power is $66 \mathrm{~kW}$ when a nominal power of the power station is 300 $\mathrm{kW}$ (22\% of the rated power of the station) [7]. The operation of the power system in this mode is accompanied by a significant increase in the specific fuel consumption relative to the nominal mode and reduction of the quality of electricity. Harmonic analysis of the load diagram, performed by the method of fast Fourier transform for aperiodic function, provides infinite series of harmonics. The most significant harmonics from that series are concentrated in the frequency range from 0 to $0.8 \mathrm{~Hz}$ (Fig. 2). To prove this, Fig. 4 shows the load diagram without harmonics above $0.8 \mathrm{~Hz}$. It basically repeats the shape of the initial load diagram that contains a full spectrum of harmonics.

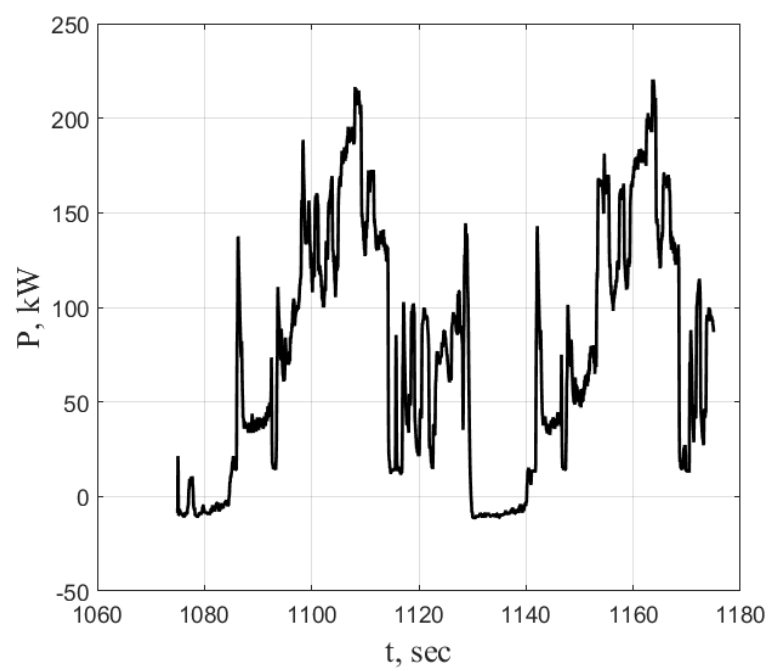

Fig. 3. Fragment of load diagram of autonomous power station of floating crane

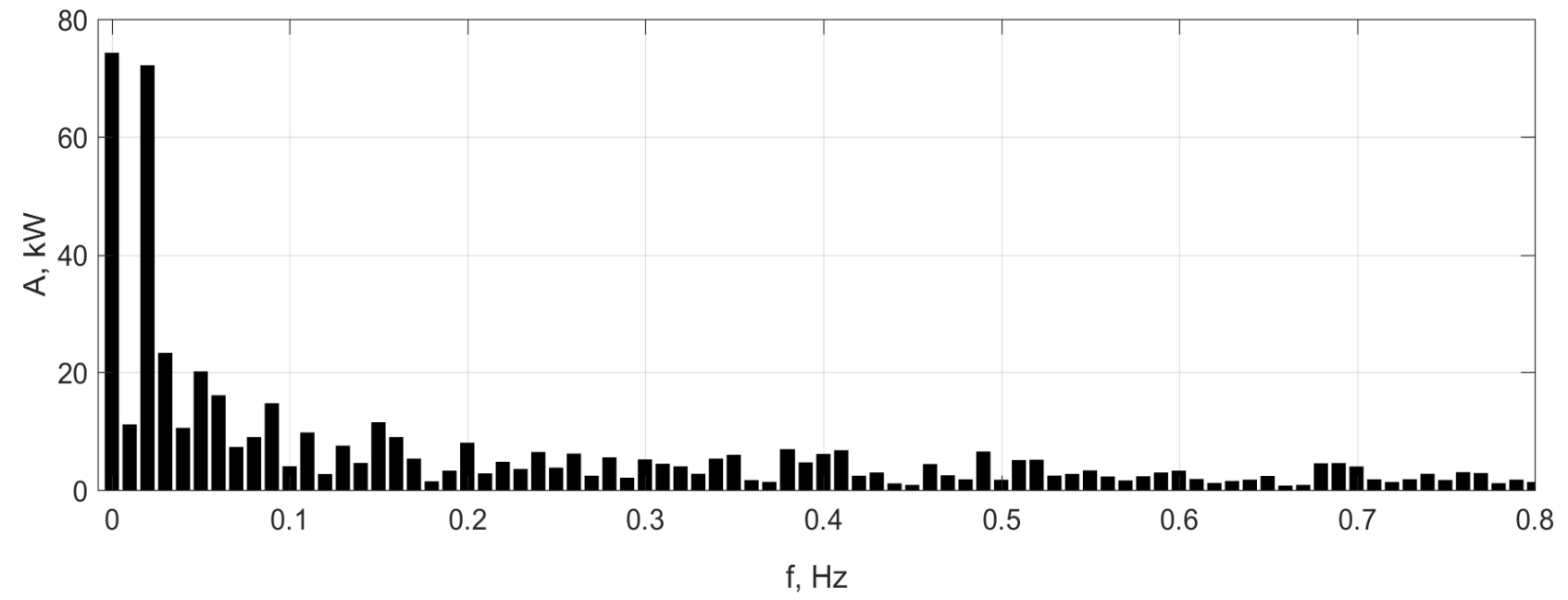

Fig. 2. Frequency response of floating crane's load diagram 


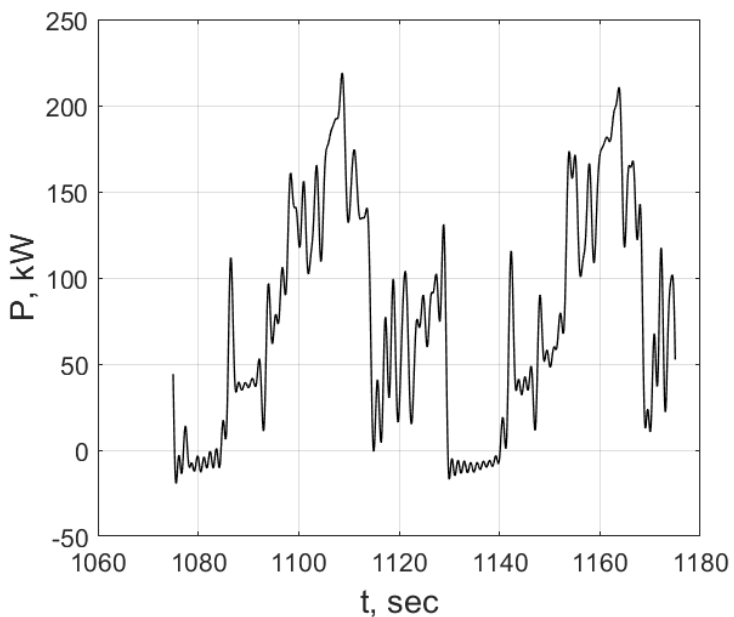

Fig. 4. Load diagram without harmonics above $0.8 \mathrm{~Hz}$

The frequency range from 0 to $0.8 \mathrm{~Hz}$ mainly matches with the frequency range of the highest sensitivity of the generators (Fig. 1) and with the LFO range $(0.1-2.0 \mathrm{~Hz})$. This increases the risk of the emergence and amplification of low-frequency oscillations. A similar load character is general for all lift and transport mechanisms of cyclic action. From Fig. 3 one can see that in the autonomous power system, the average load of the generator unit is usually $20-25 \%$ of the nominal power. The operation of the power system in this mode is accompanied by a significant increase in the specific fuel consumption relative $(45 \%)$ to the nominal mode and reduction of the quality of electricity.

\section{A TECHNIQUE FOR CHOOSING ESS PARAMETERS}

The results of harmonic analysis allow calculating parameters of energy storage depending on the amplitudes and frequencies of harmonics that should be suppressed. One of the possible strategies for using the energy storage in the case of abruptly variable load is to replace the existing generator unit with a unit, which power close to the average power of the load diagram. In this case, the control of the variable part of the load diagram must be performed by the energy storage device. It contributes to achieving complex effect: the minimum fuel rate, the minimum cost of the generator unit, the minimum costs for its servicing, the increase of the motor drive resource of the drive engine, and the reduction of losses in the generator caused by transient processes due to the alternating load. To solve the task, it is necessary to choose the power and energy capacity of the energy storage device. The minimum required power of energy storage is determined from the load diagram (Fig. 3) as the largest deviation from average value. In this case, $P=160 \mathrm{~kW}$. The diagram of energy changing was built to determine energy storage capacity (Fig. 5). According to that diagram, storage should perform energy exchange with the power system to maintain constant power of the generator. The maximum amplitude of the energy diagram shows the necessary minimum of the exchange energy value (energy of EES that should be exchanged with the power system during control). Determining the full energy capacity of energy storage is possible only after selecting the type of storage. The choice is made taking into account the specific features of the specific energy storage technology, which determines the permissible degree of charge / discharge of the storage element while maintaining a sufficient amount of available power. According to the diagram (Fig. 5), the exchange capacity of energy storage should not be less than $0.8 \mathrm{kWh}$ or $2.88 \mathrm{MJ}$. In general, if there is a necessity to damp a certain harmonic or group of harmonics in the load diagram with the use of ESS, a simple procedure must be performed for selecting the required drive parameters. For example, it is required to calculate capacity and power capacity of the store of energy for suppression of some harmonics in a load diagram in Table I.

First, based on the amplitude-frequency characteristic of the load diagram, the power of the selected harmonics is plotted against time (Fig. 6). The minimum required power of ESS is determined by the largest deviation of the power curve from the zero value. In this case, ESS power is $\mathrm{P}=60 \mathrm{~kW}$. Based on the power diagram for selected harmonics, a diagram of energy change of ESS is constructed (Fig. 7). The value of the exchange capacity of energy storage is found from the diagram of ESS energy changing for selected harmonics as maximum amplitude. In this case, ESS exchange capacity is $0.21 \mathrm{kWh}$ or $0.756 \mathrm{MJ}$. The proposed technique allows determining the ESS parameters for compensation of the selected harmonics in the load power oscillations. Fig. 8 shows the load diagram of a floating crane, where selected harmonics is excluded.

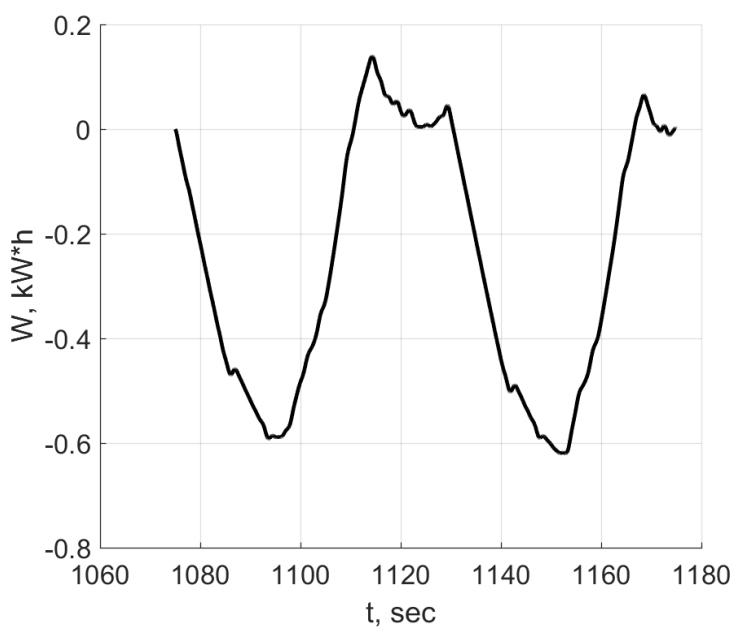

Fig. 5. Diagram of ESS energy changing

TABLE I. HARMONICS FOR SUPPRESSION

\begin{tabular}{|l|l|}
\hline \multicolumn{1}{|c|}{ Frequency of harmonic, $\mathrm{Hz}$} & Amplitude of harmonic, $\boldsymbol{k W}$ \\
\hline 0.03 & 23.2 \\
\hline 0.04 & 10.5 \\
\hline 0.05 & 20.1 \\
\hline 0.06 & 16.0 \\
\hline 0.07 & 7.3 \\
\hline 0.08 & 9.0 \\
\hline 0.09 & 14.7 \\
\hline
\end{tabular}




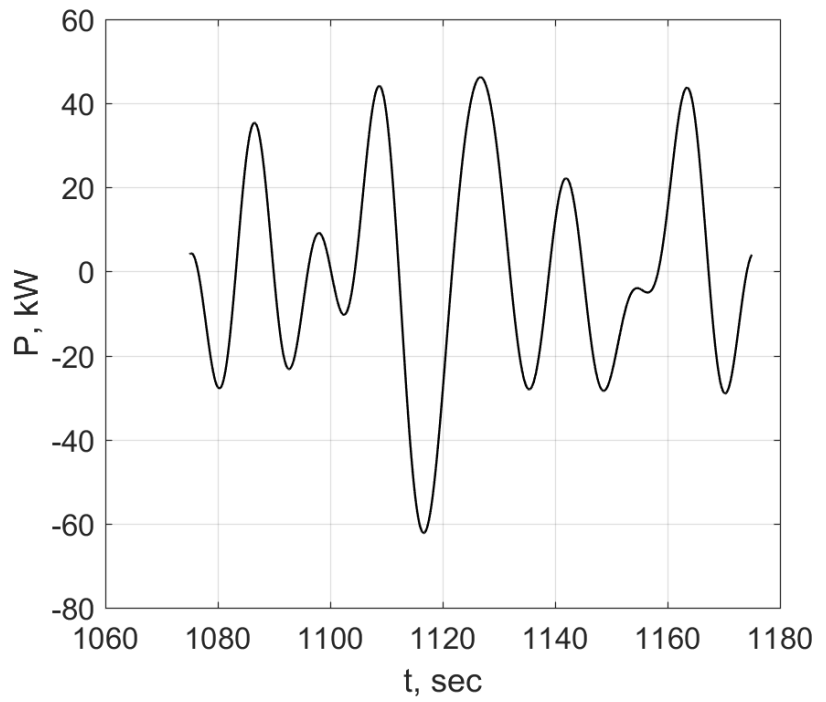

Fig. 6. Power diagram for selected harmonics

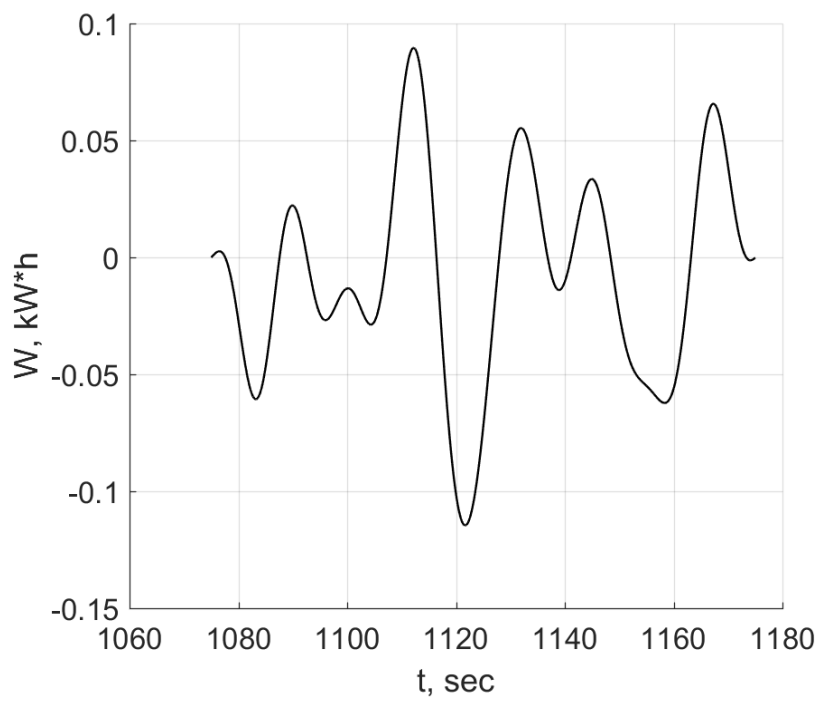

Fig. 7. Diagram of energy change of ESS for selected harmonics

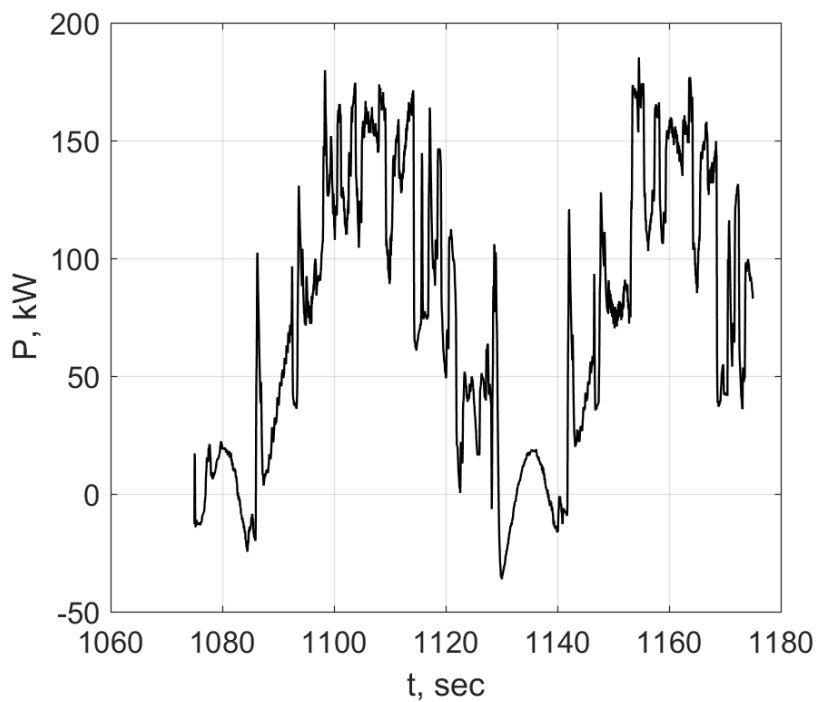

Fig. 8. Load diagram of floating crane without selected harmonics
If there is a need to remove all harmonics from the load diagram using ESS, except the main with the frequency of $\mathrm{f}=$ $0.02 \mathrm{~Hz}$, the power diagram of all harmonics for filtration must be constructed (Fig. 9). The minimum ESS required power is determined from diagram $\mathrm{P}=120 \mathrm{~kW}$. Then the diagram of ESS energy changing for filtered harmonics is calculated (Fig. 10). It determines the amount of exchange capacity of energy storage of $0.48 \mathrm{kWh}$ or $1.73 \mathrm{MJ}$. The load diagram of the generator will contain only the fundamental harmonic of power with the use of such ESS (Fig. 11).

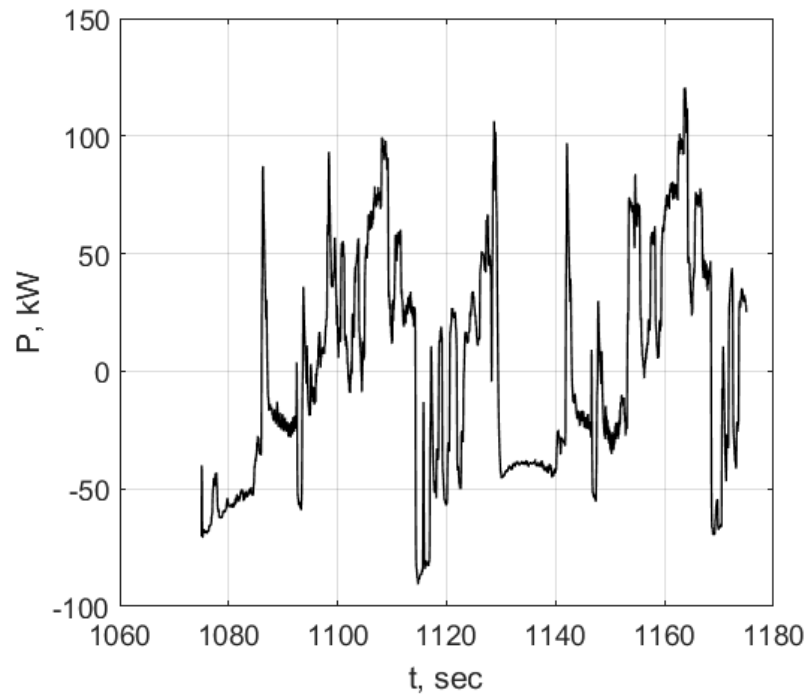

Fig. 9. Load diagram of harmonics for filtration

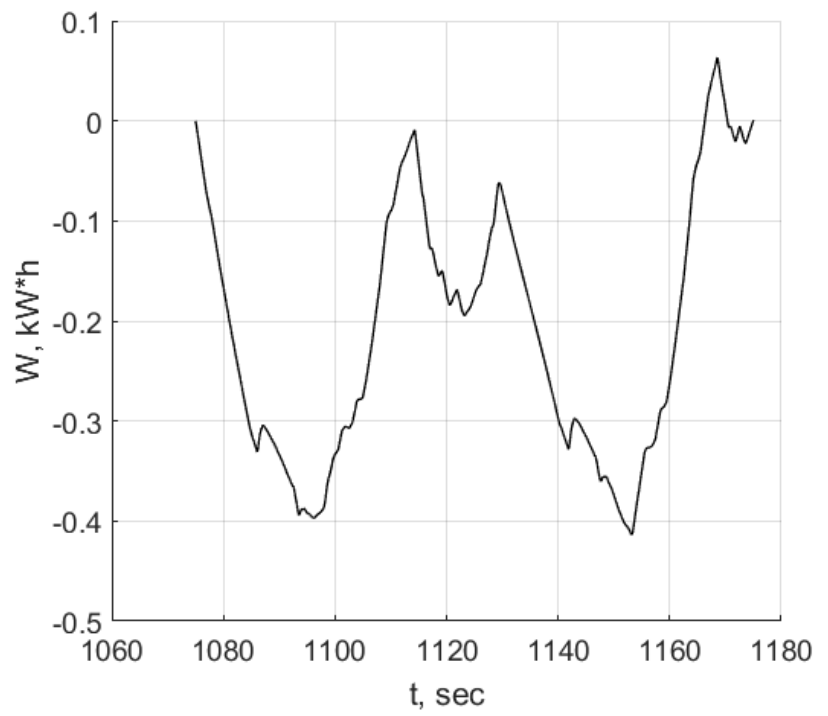

Fig. 10. Diagram of ESS energy changing for filtered harmonics

\section{Calculations}

The mathematical model was created with a help of MATLAB/Simulink (Fig. 12), and it was used to study the effectiveness of the energy storage device for damping load power fluctuations and to develop control algorithms. The model consists of a diesel generator, a load, and an energy storage model [8-10]. The generator unit consists of a diesel engine with a synchronous generator on the shaft. The unit is 
equipped with standard automatic speed and excitation regulators. The nominal voltage of the generator is $6 \mathrm{kV}$.

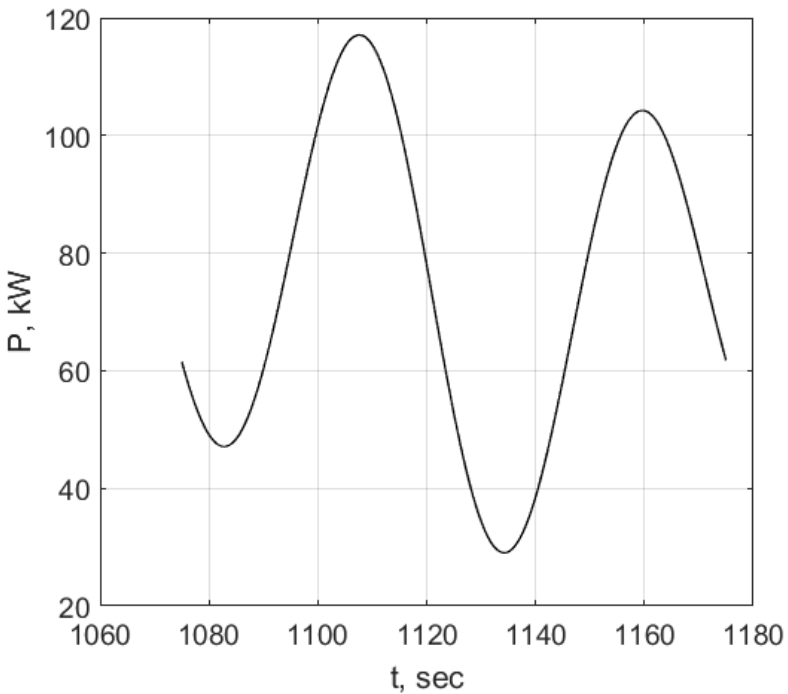

Fig. 11. Load diagram of generator after filtering

As an example, the calculation of transient processes in an isolated power system with an abruptly variable load is given for two variants of operation: without and with an energy storage device. The generator power is $80 \mathrm{kVA}$. The active load consists of three parts: a constant component of $20 \mathrm{~kW}$ and two commutated loads of $15 \mathrm{~kW}$ each. The abruptly variable load was simulated by switching on and off the two load stages (Fig. 13). Changes in the load lead to oscillations in the frequency in the power system (Fig. 14). An energy storage device based on a supercapacitor was used for load oscillations damping in the computational scheme. The parameters of the supercapacitor: capacity is $100 \mathrm{~F}$, nominal voltage is $48 \mathrm{~V}$, and a maximum power is $25 \mathrm{~kW}$. ESS is connected to the grid through a three-phase transformer with rated voltages $6 / 0.2 \mathrm{kV}$. The main parameters of the energy storage operation under the abruptly variable load damping regime are shown in Fig. 15.

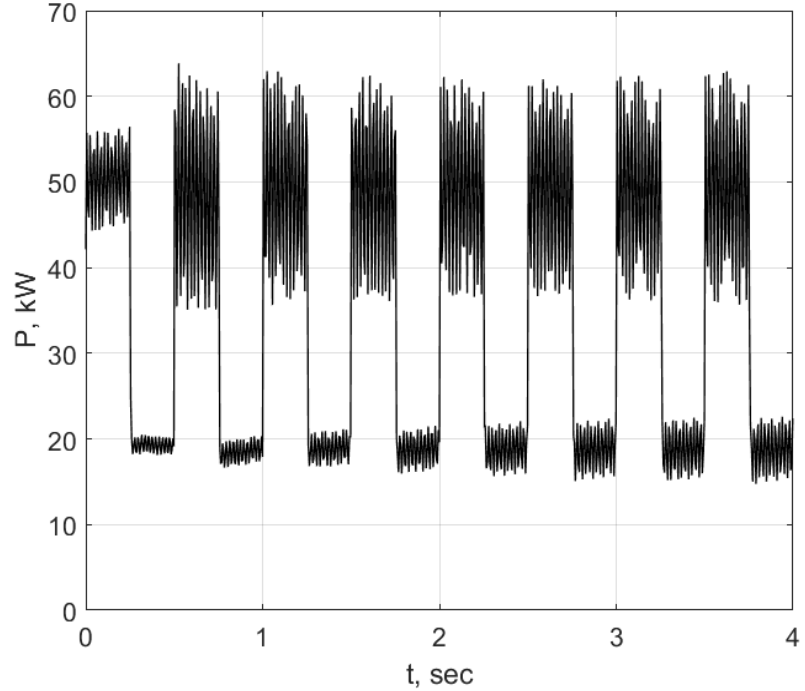

Fig. 13. Abruptly variable load schedule

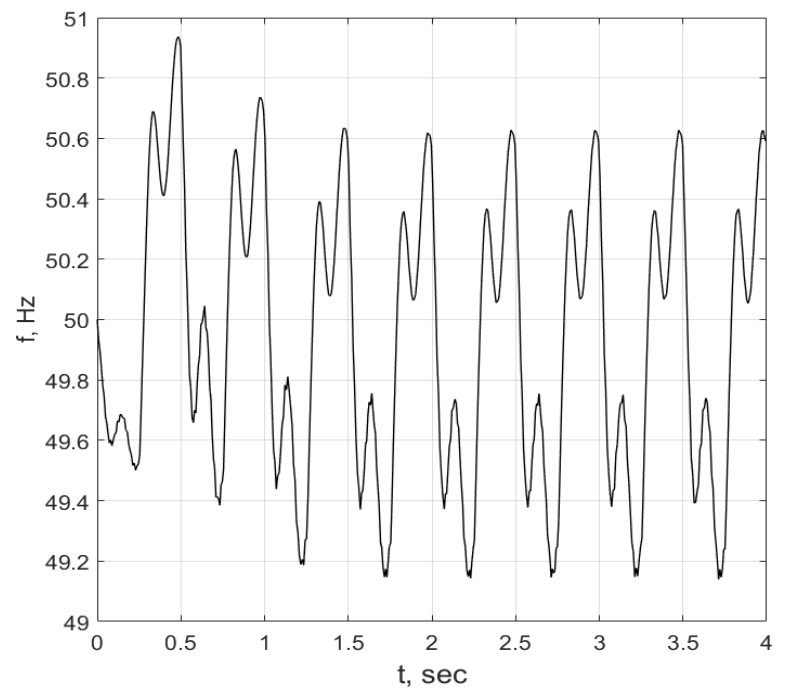

Fig. 14. Frequency of isolated system without using ESS

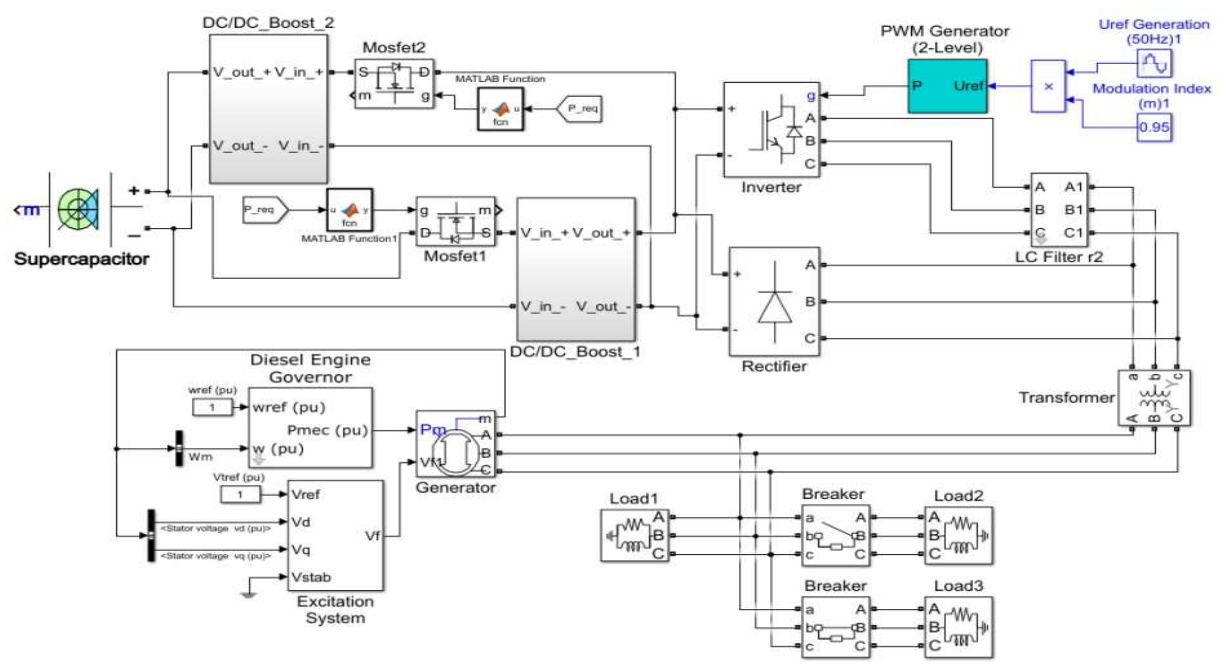

Fig. 12. Model of isolated power system in MATLAB / Simulink 


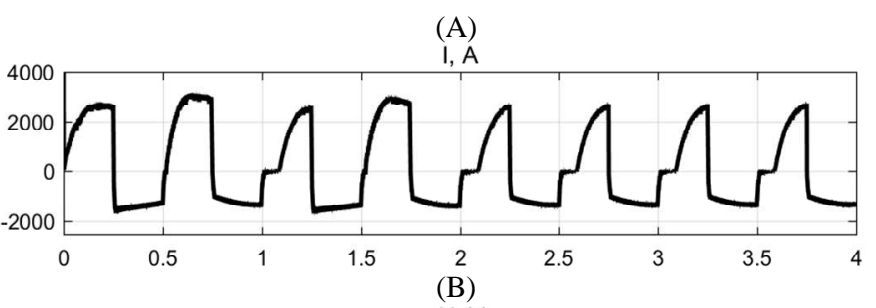

(B)

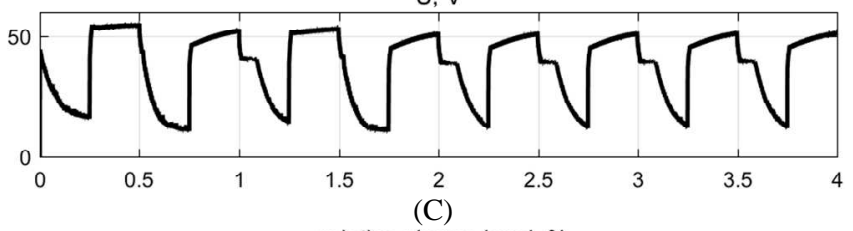

relative charge level, \%

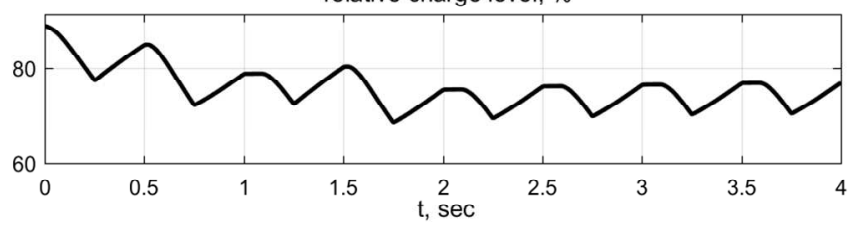

Fig. 15. Dependence of ESS parameters on time: (A) - current, (B) - voltage, (C) - relative charge level

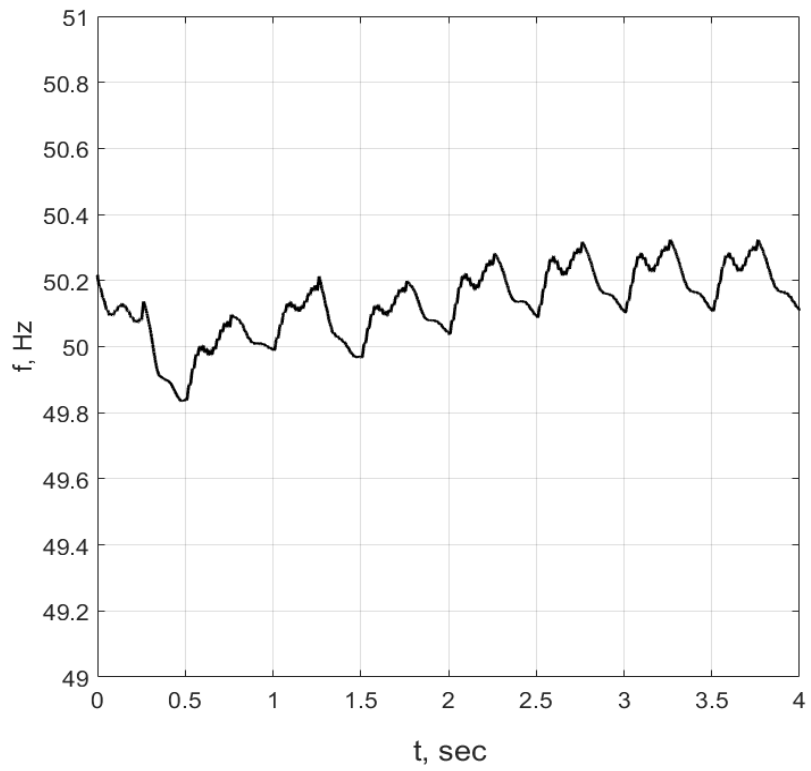

Fig. 16. Frequency of isolated system using ESS

Calculations show that the operation of ESS in the power system significantly reduces frequency oscillations occuring due to abruptly variable load (Fig. 16). The frequency amplitude is reduced by a factor of 3 due to the energy storage device actions in the considered example. At the same time, it is obvious that the quality of transients with the use of the high-speed energy storage device should be larger than in the given example. This suggests that it is necessary to synthesize an adequate system for control and developing effective algorithms and laws for controlling.

\section{CONCLUSION}

The calculations demonstrate the high efficiency of application of energy storage devices for power fluctuations damping in isolated power systems. At the same time, analysis of the results indicates that achievement of the greatest effect requires development of a methodology for optimizing the parameters of energy storage devices. A design of the control system for ESS with appropriate parameters is also an actual task. ESS is a multifunctional element of the system that can not only control active power but also can perform the functions of a reactive power control device, an active harmonic filter, and compensate for the asymmetry of the voltages of a three-phase network. This circumstance is of practical interest and requires further research.

\section{References}

[1] R.N. Berdnikov, V.E. Fortov, Ju.G. Shakarjan and K.K. Denshhikov, "Hybrid energy storage for UNPG based on batteries and supercapacitors," Energy of Unified Grid, Vol. 1, pp. 40-51, 2013.

[2] A.F. Zobaa, Energy storage technologies and applications. Rijeka, Croatia: Intech, 2013.

[3] Electric Energy Storage Systems, CIGRE Technical Brochure, Working Group C6.15, Apr. 2011.

[4] Jason C. Neely, Raymond H. Byrne, Ryan T. Elliott, C'esar A. SilvaMonroy, David A. Schoenwald, Daniel J. Trudnowski and Matthew K. Donnelly, "Damping of Inter-area Oscillations using Energy Storage," IEEE Power and Energy Society General Meeting, July 2013, DOI: 10.1109/PESMG.2013.6672775 [IEEE Power and Energy Society General Meeting (PES), 2013].

[5] T. Klimova and M. Savvatin, "Impact analysis periodically changing load on the occurrence of low-frequency oscillations," Conference proceedings, June 2015 [5th International scientific and technical conference "Actual trends in development of Power System Relay Protection and Automation", p. 953, 2015, ISBN: 978-5-9904681-2-2].

[6] Yiping Yu, Yaling Shen, Xiang Zhang, Jiyun Zhu and Jiajia Du, "The load oscillation energy and its effect on low-frequency oscillation in power system," Proceedings of the 5th IEEE International Conference on Electric Utility Deregulation, Restructuring and Power Technologies, November 2015, DOI: 10.1109/DRPT.2015.7432437 [5th IEEE International Conference on Electric Utility Deregulation, Restructuring and Power Technologies (DRPT 2015), 2016].

[7] V.A. Alemasov, A.A. Borisov and V.M. Zyryanov, "Assessment of prospects to reduce fuel consumption in the ship's power system with energy storage," Scientific problems of transportation in Siberia and the Far East, Vol. 2, pp. 215-217, 2011.

[8] Xu, N., and J. Riley. "Nonlinear analysis of a classical system: The double-layer capacitor," Electrochemistry Communications, Vol. 13, No. 10, pp. 1077-81, 2011.

[9] Doron Lifshitz and George Weiss, "Optimal Control of a CapacitorType Energy Storage System," IEEE Transactions on Automatic Control, Vol. 60, No. 1, pp. 216-220, Jan. 2015.

[10] O. Gorte, N. Kiryanova, M. Khmelik, A. Arestova, D. Baluev, G. Prankevich and V. Markin, "Assessment of energy storage effect into automatic reclosing in Smart Grid," IYCE 2015 - Proceedings, May 2015, Art. 7180757, DOI: 10.1109/IYCE.2015.7180757 [5th International Youth Conference on Energy (IYCE), 2015]. 